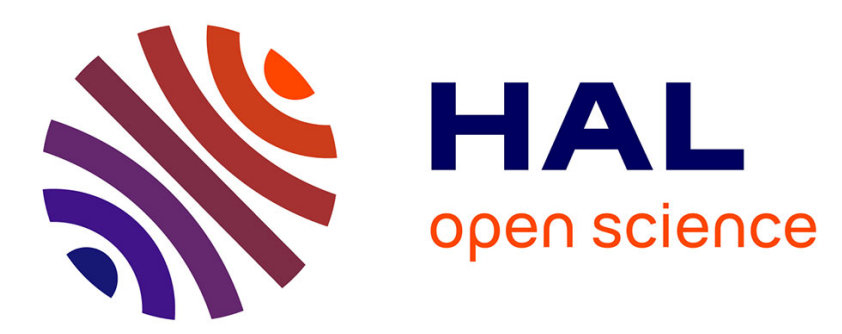

\title{
Vers la fin d'une règle légendaire: concordance des temps et non-concordance modale en espagnol moderne
}

Gabrielle Le Tallec Lloret

\section{To cite this version:}

Gabrielle Le Tallec Lloret. Vers la fin d'une règle légendaire: concordance des temps et nonconcordance modale en espagnol moderne. Langages, 2013, La concordance des temps: vers la fin d'une règle ?, 191. halshs-01420748

\section{HAL Id: halshs-01420748 \\ https://shs.hal.science/halshs-01420748}

Submitted on 21 Dec 2016

HAL is a multi-disciplinary open access archive for the deposit and dissemination of scientific research documents, whether they are published or not. The documents may come from teaching and research institutions in France or abroad, or from public or private research centers.
L'archive ouverte pluridisciplinaire HAL, est destinée au dépôt et à la diffusion de documents scientifiques de niveau recherche, publiés ou non, émanant des établissements d'enseignement et de recherche français ou étrangers, des laboratoires publics ou privés. 


\title{
Vers la fin d'une règle légendaire : concordance des temps et non-concordance modale en espagnol moderne
}

\author{
Gabrielle LE TALLEC-LLORET \\ Université Rennes 2
}

\begin{abstract}
Résumé
Pour tout hispaniste français la règle de la «concordance des temps » (CDT) est une règle d'or, et fait partie de ces «normes » traditionnelles tyranniques, solidement ancrées dans l'enseignement de la syntaxe, alors que leur pertinence au regard de l'histoire de la langue ou de la réalité linguistique de l'espagnol n'a jamais été scientifiquement établie. Dans la tradition grammaticale et linguistique espagnole, l'étude de la CDT est un classement des effets de discours des temps verbaux, assimilant temps verbal et temps vécu - le temps verbal appelé «présent » qu'il soit classé dans le mode «indicatif » ou dans le mode «subjonctif » fait toujours référence au temps présent de l'expérience -, et adoptant une méthode clairement référentialiste.

Une autre approche du phénomène est possible avec la nouvelle théorie des modes et des temps de Gilles Luquet, en particulier l'opposition entre un mode actualisant et un mode inactualisant, fondée sur l'observation du signifiant des formes verbales. Cette théorie, outre l'opportunité qu'elle offre de résoudre les difficultés qu'engendre la traditionnelle opposition entre mode « indicatif » et mode «subjonctif», permet de rendre compte de tous les emplois, y compris ceux qualifiés de «discordants », et de comprendre les mécanismes à l'œuvre dans le choix d'une forme ou d'une autre. Au sein du mode inactualisant, la langue espagnole ne contraint à aucun respect ou non-respect de la concordance des temps, mais elle autorise l'alternance en fonction du degré d'inactualité retenu.
\end{abstract}

Mots-clés : concordance des temps, référentialisme, chronologie relative, dislocation des temps, système, unité du signe, signifiant, modes, subordination, actuel, inactuel.

\begin{abstract}
For every French hispanist the "sequence of tenses" (SOT) " rule is a golden rule, and belongs to these traditional tyrannical "norms", deeply rooted in the teaching of syntax, although their relevance from the history of language or the linguistic reality of Spanish point of view has never been scientifically established.In the Spanish grammatical and linguistic tradition, the study of SOT is a classification of the discourse effects of verbal tenses, assimilating verbal tense and lived tense -the verbal tense called "present", either classified in the "indicative" mode or in the "subjunctive" mode is always referring to the present time of the experience-, and adopting clearly a referencialist method. Another approach of the phenomenon is possible with the new theory of the modes and tenses by Gilles Luquet, in particular the opposition between an actualizing mode and an unactualizing mode, based on the observation of the signifiant of the verbal forms. This theory, besides the opportunity it is offering to solve the difficulties generated by the traditional opposition between "indicative" mode and "subjunctive" mode, allows to report all uses, including those as "discordants", and to understand the mechanisms in process in the choice of a form or another. Within the inactualizing mode, the spanish language does not constrain to any respect or non respect of the SOT, but authorizes the alternation according to the chosen degree of inactuality.
\end{abstract}

Keywords: sequence of tenses, referencial, ,, relative chronology, tense dislocation, system, sign unity, significant,mode, subordination, actual, unactual. 


\section{Introduction}

S'il est une règle absolument incontournable pour tout francophone faisant l'apprentissage de la langue espagnole dans les grammaires françaises, c'est bien celle de la « concordance des temps ». Cette règle de la syntaxe espagnole commande un lien de dépendance présenté comme automatique, presque mécanique, entre le «temps » du verbe de la proposition principale, au mode indicatif, et celui du verbe de la proposition subordonnée, au mode subjonctif, «si l'action exprimée est considérée comme non réalisée ou non réalisable » (Gerboin \&Leroy: 224). La règle préconise l'application de la mécanique temporelle suivante :

Proposition principale à l'indicatif (présent et passé composé, futurs simple et antérieur) ou à l'impératif $\rightarrow$ Proposition subordonnée au subjonctif présent.

1. Le piden a Pablo que estudie más

Proposition principale à l'indicatif (imparfait, plus-que-parfait, prétérit, passé antérieur, conditionnels présent et passé) $\rightarrow$ Proposition subordonnée au subjonctif imparfait.

2. Le pidieron a Pablo que estudiara más

Bien entendu, ne répondent à cette règle que certains effets de sens, les autres étant qualifiés de «transgressifs » en particulier la combinaison d'un prétérit dans la principale avec un subjonctif présent dans la subordonnée :

\section{Le pidieron a Pablo que estudie más}

Présentée comme une transgression, la combinaison pidieron estudie est justifiée par la valeur «temporelle » qu'auraient les deux formes de subjonctif, l'une à l' " imparfait », l'autre au " présent ». Les deux événements déclarés par ces subjonctifs, estudiara et estudie, sont présentés comme s'opposant temporellement, du point de vue de l'expérience, de la chronologie des actions qu'elles servent à évoquer, et non du point de vue de la représentation temporelle qu'elles véhiculent. En résumé, un subjonctif « présent » évoque une action présente ou future, un subjonctif «imparfait» une action passée. Avec estudiara l'action appartient au passé, tandis qu'avec estudie, l'action a des effets dans le présent. D'un côté on tire vers le passé du locuteur, pris comme repère, du fait du temps de la principale, et l'on justifie ainsi le subjonctif imparfait, canonique, pour faire concorder deux actions dans le passé ; de l'autre, on tire vers le présent ou « l'avenir » du locuteur, toujours à partir du point de repère dans le passé (temps de la principale) pour justifier un cas de non-concordance (prétérit présent) par une non-concordance dans le temps événementiel.Ainsi, on met sur le même plan les temps verbaux du mode indicatif et ceux du mode subjonctif, et l'on assimile pour les deuxle « temps » linguistique - «présent », «prétérit », « imparfait » - et la chronologie des événements : passé, présent, futur, autour de la figure du locuteur. 


\section{Fausse norme et vrai usage}

La grammaire française de Gerboin et Leroy est emblématique d'une démarche très largement adoptée ${ }^{1}$ : la mise à l'écart de nombreux emplois pourtant autorisés par le système linguistique. Que le rejet soit implicite - manuels du secondaire, ouvrages grammaticaux de grande diffusion, grammaires de Bouzet, Coste et Redondo -, ou explicite - grammaires de Bedel, Ligatto et Salazar -, de nombreuses combinaisons sont présentées comme déviantes, au sein d'un exposé toujours clairement hiérarchisé : usage canonique versus usage non canonique, ou sa variante, usage courant versus usage marqué.

Bien d'autres combinaisons sont possibles, mais elles ne sont prises en compte ni dans le cadre simpliste et restrictif de la « règle de la concordance des temps », puisqu'elles la contredisent, ni dans celui des exceptions. Ces combinaisons, autres, sont tout simplement occultées afin de justifier une règle ne retenant qu'une partie des combinaisons observables dans le discours.Pourtant, le subjonctif «présent » de la subordonnée peut être combiné, dans la principale, notamment avec le prétérit, l'imparfait de l'indicatif

(1) Al principio yo escuchaba las penas y dejaba que lloren sobre mí y me llenaba de lágrimas y por eso era medio barrigona [...] (Pequeñas resistencias 3. Antología del nuevo cuento sudamericano, 2004, Madrid : Página de espuma, p. 120),

le plus-que-parfait

(2) La calma vuelve hoy a las calles de Georgia tras las eufóricas celebraciones de las últimas horas por el triunfo de la revuelta popular, que ayer logró tumbar a Shevardnadze sin una gota de sangre. Tanto Estados Unidos como la Unión Europea (UE) mostraron su apoyo a Buerdzhanadze, pero le habían exigido que celebre cuanto antes unas elecciones legislativas de acuerdo con la Constitución para legitimar los cambios en la cúpula del poder. (El País, 24-11-03),

le conditionnel présent

(3) Por esto resultaría chocante la posposición de un adjetivo que signifique cualidades inseparablemente asociadas a la imagen del sustantivo [...] (RAE, Esbozo de una nиеva Gramática de la lengua española, 2004, Madrid : Espasa, p. 410)

le conditionnel passé

(4) El PSC dice que le habría gustado que su lista para asambleístas esté presidida por Jaime Nebot, pero no podrá ser porque es candidato presidencial. (Diario Hoy, 03-09-97)

Par ailleurs, le subjonctif imparfait peut-être combiné, notamment, avec un verbe au présent de l'indicatif dans la principale,

(5) Lo vi por segunda vez dos días después, saliendo de los Tribunales. Yo estaba bajando la escalinata de mármol de la entrada y veo un tipo en mangas de camisa esperando junto a un taxi

\footnotetext{
${ }^{1}$ Pour une critique de cette approche, voir G. Luquet (2007 : 44) et G. Le Tallec-Lloret (2010b : 63-73)
} 
que bajara un pasajero que en ese momento le estaba pagando al conductor, (J. José Saer, 2003, Cicatrices, Buenos Aires : Seix Barral, p. 74),

etmême avec un verbe au futur antérieur de l'indicatif :

(6) Es difícil que podamos imaginar cómo habrándeseado muchos corredores que este Giro alcanzara su final. Llegar a Milán ha sido para muchos un sueño no cumplido, para los supervivientes ha supuesto la inmensa alegría de culminar el Giro más duro [...] (El Mundo, 1306-94)

\section{La référence à l'univers au cœur du processus explicatif}

Dans la tradition grammaticale et linguistique espagnole, l'approche de la «concordance des temps» est uniquement de type référentialiste. On ne s'intéresse pas à la nature des temps verbaux organisés en système, mais exclusivement à ce à quoi ils renvoient ${ }^{2}$.

C'est le parti-pris de la référentialité qui sous-tend entièrement l'étude d'Á. Carrasco Gutiérrez, « La concordancia de tiempos en las gramáticas del español» (1994 : 113-131), où elle rend compte des différentes approches du «mode subjonctif » et du phénomène de la «concordance des temps »dans la tradition grammaticale espagnole. Ellepasse en revue études linguistiques et grammaires espagnoles depuis l'édition de la Real Academia de 1796 jusqu'aux études linguistiques de Rojo (1976) et Veiga (1987, 1991).

Concordance des temps est le nom désignant le phénomène grammatical selon lequel l'indication temporelle apportée par le verbe de la proposition subordonnée substantive n'est pas sans rapport avec l'indication temporelle apportée par le verbe de la proposition principale, conséquence de la relation de dépendance syntaxique qui existe entre eux. (p. 113. Nous traduisons)

L'approche de la CDT a toujours comme cadre syntaxique une proposition subordonnée substantive, qu'elle soit au «mode indicatif» ou au «mode subjonctif ». L'opinion communément admise $^{3}$ pour les subordonnées au subjonctif est la suivante : la CDT se définit comme « le phénomène grammatical selon lequel le temps du verbe principal et le temps du verbe subordonné appartiennent à la même sphère temporelle ».

La méthode est strictement la même dans le chapitre de la Gramática descriptiva de la Lengua Española(1999), consacré à la CDT : «El tiempo verbal y la sintaxis oracional. La consecutio temporum » (p. 3061-3128), rédigé par Á. Carrasco Gutiérrez.

Ce phénomène renvoie à la relation de dépendance qui s'établit entre les interprétations temporelles des deux formes verbales si entre leurs propositions respectives il existe aussi une relation de dépendance ou de subordination syntaxique. (p. 3063. Nous traduisons)

\footnotetext{
${ }^{2}$ Pour une critique détaillée de cette approche, voir G. Le Tallec-Lloret (2010b : 24-72)

${ }^{3}$ Real Academia (1976), Salvá (1830), Martínez Sevilla (1851), Real Academia (1858, 1880), Commelerán y Gómez (1881), Pérez Barreiro (1897), Burgos Lanchares (1905), Cejador (1905), Sanmartí (1907), Hanssen (1913), Lemus y Rubio (1920), Lenz (1920), Robles Dégano (1924), Real Academia (1931), Gili Gaya (1943), Seco (1954), Farley (1965), Obaid (1967), Farley (1970), Fernández Álvarez (1972), Suñer (1979), Padilla Rivera (1985), Borrego, Asencio y Prieto (1986) Suñer y Padilla Rivera (1987), Suñer (1990).
} 
Un tel parti-pris, celui de la «chronologie relative » aboutit à un classement de l'interprétation des temps verbaux - c'est-à-dire du temps d'événement-, proposé par la Gramática descriptiva, un chapitre plus loin, en ne retenant que les combinaisons qui laissent voir une certaine concordance de formes : « le temps de l'événement subordonné peut être antérieur au temps de l'événement principal, postérieur ou simultané ».

Le temps principal appartient à la sphère du présent.

Me extraña que Juan se haya callado hasta ahora (antériorité)

Insistimos en que te encargues de todo de ahora en adelante (postériorité)

Es imposible que ya lo hayas hecho (simultanéité)

Le temps principal appartient à la sphère du passé.

El testigo ha negado que le hubieran ofrecido dinero por cambiar su declaración (antériorité)

Yo he querido que mantuviese viva siempre la memoria de lo que pasó (postériorité)

Le sorprendió que el camión de la basura pasara los domingos (simultanéité). (p. 3082-3083)

Le classement des valeurs d'effet cherche à décrire comment le temps de la subordonnée au subjonctif pose des événements à une époque déterminée relativement au temps de la principale à l'indicatif.Par rapport au temps du verbe de la principale, trois relations temporelles sont susceptibles d'être exprimées par le temps du verbe de la subordonnée : antériorité, postériorité, simultanéité.

En suivant cette méthode, on aboutit à cette incohérence où la combinaison des deux mêmes formes verbales (présent/subjonctif passé) donne lieu à deux interprétations différentes et, en outre, fort discutables :

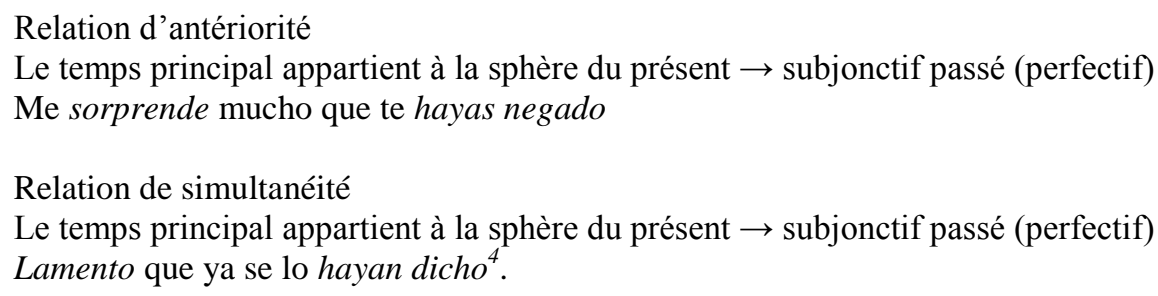

On confond ce à quoi servent les temps verbaux, ce qu'ils permettent d'exprimer dans un énoncé particulier, combinés à d'autres informations linguistiques, et leur valeur puissantielle, constante, au sein d'un système. Si la subordonnée est au mode indicatif, on veut bien admettre que le temps du verbe subordonné est de même nature, c'est-à-dire permet le même type de repérage temporel que celui du verbe de la principale, à partir du présent d'énonciation. Par rapport au temps de la proposition principale, à l'indicatif, le locuteur pourra effectivement choisir trois rapports temporels : une relation d'antériorité, une relation de postériorité, une relation de simultanéité. On pourra alors parler de « concordance des temps » à l'intérieur du mode indicatif.

\footnotetext{
${ }^{4}$ Nous ne voyons pas de relation de simultanéité dans l'exemple « Lamento que ya se lo hayan dicho ».
} 
En revanche, si la subordonnée est au subjonctif, il nous semble très critiquable, dans une approche linguistique, non seulement d'aligner sémantiquement le «temps» dans le mode subjonctif sur le «temps» dans le mode indicatif, mais surtout de ne pas mentionner bien d'autres combinaisons permises par le système. etd'occulter l'une des caractéristiques du système verbal espagnol : la possibilité de contraste offerte au locuteur.

Ce classement des effets de sensn'aboutit qu'à l'énonciation d'une seule restriction - celle liée à la nature sémantique du verbe subordonnant ${ }^{5}$-, et à l'inscription, dans le signifié de langue des formes verbales, de «valeurs » qui ne sont que contextuelles, pour tenter de justifier certains emplois jugés marginaux ou non canoniques ${ }^{6}$.

\section{Non-concordance modale : mode actualisant mode inactualisant}

Sur le plan morphologique, le système verbal espagnol distingue des formes pourvues de flexions personnelles, d'un côté, et des formes qui en sont dépourvues, de l'autre. C'est ce première critère, la personne, que retient Gilles Luquet dans sa nouvelle théorie des modes, La teoría de los modos en la descripción del verbo español.Un nuevo planteamiento (2004).À partir d'un second critère, le rapport entre exochronie et endochronie, G. Luquet opère un premier partage entre deux sous-ensembles de formes, l'un, non personnel, apte à livrer des informations sur le seul temps porté ; l'autre, personnel, apte à livrer des informations sur le seul temps portant :

[...] - un sous-ensemble de formes dont le propre est de ne fournir que des représentations possibles du temps $t$ impliqué par une opération. La nature du temps dans lequel s'inscrit ladite opération - le temps $T$ - reste, dans chacune de ces formes, indéterminée ;

-un sous-ensemble de formes dont le propre est de ne fournir que des représentations possibles du temps $T$ dans lequel s'inscrit une opération. C'est la nature du temps intérieur à l'opération - le temps $t$-qui reste, dans ces autres formes, indéterminée.

Le premier de ces sous-ensembles est constitué de formes non personnelles. Le deuxième est constitué de formes personnelles. (2007: 46)

Ces deux variations structurantes (personne et rapport exochronie/ endochronie), amène G. Luquet à constater que dans les formes personnelles, il n'existe pas, à proprement parler, de mode subjonctif sur le plan du signifiant :

Les seuls traits de sémiologie que partagent un «présent», un «futur » et un «imparfait» du subjonctif - en l'occurrence les marques de la personne - sont de ceux que l'on trouve également dans d'autres « temps » de la conjugaison espagnole et notamment dans certains temps du mode appelé «indicatif. Il en est ainsi de l'absence de marques spécifiques concernant les $1^{\mathrm{e}}$ et $3^{\mathrm{e}}$ personnes du singulier; [...] du $-s$ terminal de la $2^{\mathrm{e}}$ personne du singulier ; [...] des morphèmes terminaux -mos, -is et $-n$, chargés de représenter respectivement les $1^{\mathrm{e}}, 2^{\mathrm{e}}$ et $3^{\mathrm{e}}$ personnes du pluriel.

\footnotetext{
${ }^{5}$ Pour une critique de cette approche chez Kowal (2007) et Varga (1998), voir G. Le Tallec-Lloret (2010b : 60-62)

${ }^{6}$ Sur la confusion entre signifié et référence dans les théories de la «Dislocación de los tiempos » (A. Veiga et G. Rojo 1999) et celle de l' «Interpretación de doble acceso » d' Á. Carrasco Gutiérrez (2000) reprenant la théorie de M. Enç, « Anchoring conditions for tense » (1987), voir G. Le Tallec-Lloret (2010b : 48-55).
} 
Ces marques-là ne sont pas spécifiquement subjonctives puisqu'on les trouve aussi bien dans la structure signifiante de n'importe quel imparfait de l'indicatif ou de n'importe quel conditionnel. Quant aux morphèmes thématiques auxquels elles s'adossent, ils n'ont rien, du point de vue sémiologique, qui oblige à les regrouper dans un seul et même ensemble. (1998:89-90)

Il existe, en revanche, une opposition franche entre 3 formes verbales personnelles non auxiliées distinguant les personnes 1 et 3 de la conjugaison, et les autres, qui neutralisent cette distinction.

À partir de ces trois observations de surface, et contrairement à la tradition guillaumienne qui voyait dans le «présent $» d u$ mode indicatif un aboutissement ${ }^{7}$ de l'opération chronogénétique, G. Luquet fait du présent d'énonciation la source du système verbo-temporel. Par "présent» d'énonciation, il faut comprendre LE présent unique de l'être qui se définit sous l'espèce du MOI, à la fois locuteur et constructeur du langage ${ }^{8}$. C'est en tant qu'instance énonciatrice que le MOI se construit ses représentations du temps ou images-temps. Les formes personnelles et impersonnelles représentent différentes conceptions du temps associé à une opération : c'est cette conception du temps que l'on appelle le temps linguistique. Dans les formes personnelles, on distinguera deux types de repérages autour de la figure du locuteur, clé de voûte de cette architecture temporelle.

-Un repérage réellement temporel, fondé sur le temps d'expérience du locuteur, permettant de situer dans le temps une opération.

L'une d'elles est celle qui se construit à partir du présent, c'est-à-dire à partir de l'espace temporel - éminemment singulier - dans lequel le Moi se situe lui-même et dans lequel il inscrit son activité. Associé à la représentation de ce qui «est », le présent, dans l'univers de ce Moi, s'oppose, d'une part au passé - lieu temporel de ce qui «n'est plus » - et d'autre part, au futur lieu temporel de ce qui «n'est pas encore ». Figurativement

\section{temps d'expérience}

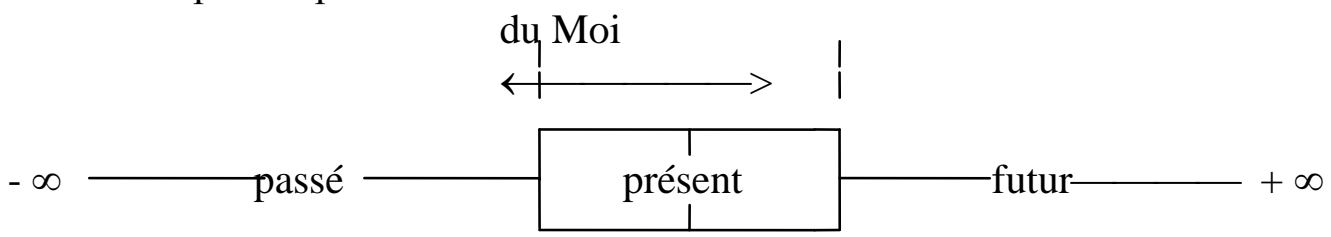

Dans un univers temporel objectivé de la sorte, le présent, par définition, a une extension subjective et variable et il doit comprendre, au minimum, deux instants de nature différente. Le présent est le lieu du temps dans lequel un instant de futur se convertit continuellement en un instant de passé : il est le lieu dans lequel se fonde, en pensée commune, la mobilité du temps. (2007 : 48)

\footnotetext{
${ }^{7}$ Voir Gérard Moignet : «Système de langue, il manifeste sa cohérence dans la progression qu'il montre d'une image du temps de plus en plus précise et différenciée à mesure que l'on va du plus virtuel au plus actuel », Systématique de la langue française, 1981, Paris, Klincksieck, p.65. Nous soulignons.

${ }^{8}$ En dehors de l'acte de langage, hors prise de parole, il existe autant de présents que d'êtres qui les conçoivent.
} 
C'est le type de repérage proposé par les formes actualisantes telles que les a définies G. Luquet: présent, prétérit et futur du mode actualisant, c'est-à-dire fondé sur un présent actualisé. Ce repérage se fondant sur le moment de locution le présent du locuteur - celui-ci peut ainsi rattacher un événement à son présent de locution en l'actualisant.

Cet univers offre donc les avantages de toute représentation construite au plus près d'une certaine expérience, mais il en a aussi les inconvénients, au nombre desquels figure l'obligation de tout y ramener à l'actualité-réalité et celle d'y loger des opérations rapportées à des êtres formellement définis par rapport au MOI. En français, comme dans toutes les langues évoluées, l'existence de plusieurs représentations linguistiques de l'univers-temps, est essentiellement un moyen d'échapper à ces contraintes. (Ferreres Maspla \& Luquet $1990: 29-30)$

Il existe bien une ligne du temps, lorsqu'elle est construite par rapport au locuteur, à son présent d'énonciation; mais dès lors que le locuteur renonce à ramener tous les événements à lui, il faut alors concevoir plusieurs «lignes », plusieurs images-temps. Au lieu du temps opératif guillaumien conduisant à l'actuel, G. Luquet propose, à partir du présent actualisé, un dégradé de l'inactuel. Le locuteur peut se dédoubler mentalement et, en tant qu'observateur, se transporter mentalement, imaginairement, dans le passé ou dans le futur; l'observateur est ainsi susceptible de se déplacer en n'importe quel lieu du temps.

-Plusieurs formes verbales réunies dans le mode inactualisantpermettent un repérage de l'inactuel, emportant une conception du temps d'une autre nature, un temps conceptuel, imaginaire, et non plus un temps d'expérience.

L'objectivation de l'univers temporel d'un sujet parlant peut cependant avoir un autre point de départ que la représentation du présent d'expérience. Elle peut se fonder, par exemple, sur la représentation d'un présent délié de toute expérience du temps, c'est-à-dire sur la représentation d'un présent fictif - un présent purement et simplement imaginaire - susceptible de coïncider ou non avec le présent d'énonciation. (2007: 48-49)

Les formes verbales inactualisantes tournent aussi autour de la figure du locuteur -ce sont des formes verbales pourvues de flexion personnelle -, mais déliées du temps d'expérience, elles appartiennent au mode de l'inactuel. Une deuxième «ligne » du temps a ainsi pour position-repère un présent délié de toute expérience du temps à partir duquel le MOI observateur se représente un événement. Le MOI observateur peut se situer imaginairement dans l'espace temporel en coïncidence avec sa prise de parole (le présent d'expérience), ou dans son avant, ou dans son après. Le locuteur-observateur aura ainsi la possibilité de se représenter dans son univers temporel, un présent imaginaire, cette fois, inactualisé et un futur inactualisé : ces deux représentations correspondent sémiologiquement à l'ex-« imparfait de l'indicatif » et à l'ex-« conditionnel », le «passé inactualisé » n'ayant pas de forme en espagnol. 


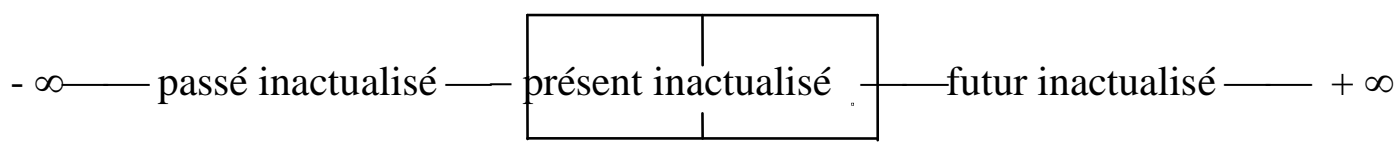

Les formes verbales se partagent non pas entre le mode «indicatif » et le mode «subjonctif» mais entre deux modes offrant deux conceptualisations du temps, l'une actualisante, l'autre inactualisante, l'une permettant au locuteur de ramener tout événement à son temps d'expérience, l'autre fondée sur l'imagination. Chacun des deux modes est conçu comme un mode de représentation mentale, intériorisée, du temps.

Dans cette conception, le temps n'est pas cette donnée objectale amenant, de façon quelque peu simpliste, à brancher directement le temps linguistique sur le temps des événements. Le locuteur se trouve bien au centre des deux sous-systèmes mais pour deux repérages de nature extrêmement différente, selon qu'il souhaite rattacher un événement à son temps d'expérience ou se donner une représentation imaginaire, abstraite, d'un événement et le détacher de son actualité.

C'est la raison pour laquelle, l'opposition entre formes actualisantes et formes inactualisantes est de nature modale, absolument incompatible avec l'opposition traditionnelle entre mode indicatif et mode subjonctif. En cela, la théorie de G. Luquet est en rupture, non seulement avec la terminologie, mais surtout avec l'organisation traditionnelle des temps verbaux que supposaient ces deux «chapeaux». Toute forme inactualisante appartient de fait au mode de l'inactualisant.

La forme en - $e /-a$ et les formes en - $r a /-s e$ appartiennent au mode inactualisant, chacune proposant un degré d'inactualité différent, exploitable par le sujet parlant en fonction de sa visée discursive.

Une autre façon d'objectiver l'univers temporel d'un sujet parlant est celle, plus abstraite encore, qui consiste à lui donner une forme simplement bipartite, c'est-à-dire la forme d'une extension en laquelle une simple limite associée à l'instant d'énonciation permet de distinguer un espace ouvert à l'activité du Moi - un 'présent-futur' indifférencié - et un espace fermé à cette activité. Figurativement :

\section{Moment \\ de l'énonciation}

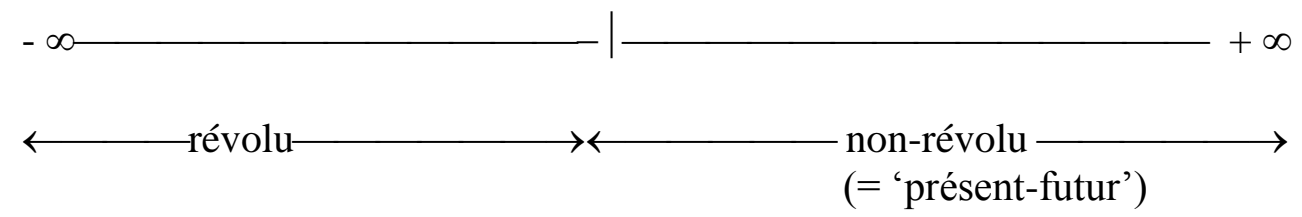

Dans le verbe espagnol - et dans son état actuel de définition - la représentation d'un 'présentfutur' indifférencié est celle que véhiculent les formes du type cante, cantes, cante... La représentation du temps révolu qui s'oppose au 'présent-futur' de ces formes n'a pas, elle, de signifiant. 
Une dernière façon d'objectiver l'univers temporel d'un sujet parlant est celle qui consiste à ne privilégier aucun de ses éléments constitutifs. C'est celle qui consiste à abstraire de cet univers la représentation de toute extension singulière et même de toute limite singulière :

Dans le verbe espagnol, la représentation de cet univers est celle que véhiculent deux séries de formes : cantara, cantaras, cantara... et cantase, cantases, cantase... Cantara et cantase sont les formes personnelles du verbe qui, en langue, inscrivent un événement dans le cadre temporel le plus abstrait que l'on puisse concevoir. (Luquet 2007 : 50)

Les formes verbales personnelles en espagnol moderne s'organisent donc en deux sous-ensembles modaux : d'un côté, un mode actualisant offrant trois types de représentations : canto, canté, cantaré ; de l'autre, un mode inactualisant offrant cinq types de représentations : cantaba, cantaría, cante, cantara, cantase.

À partir de cette théorie, nous proposons une vision du système verbo-temporel espagnol qui pourrait être la suivante :

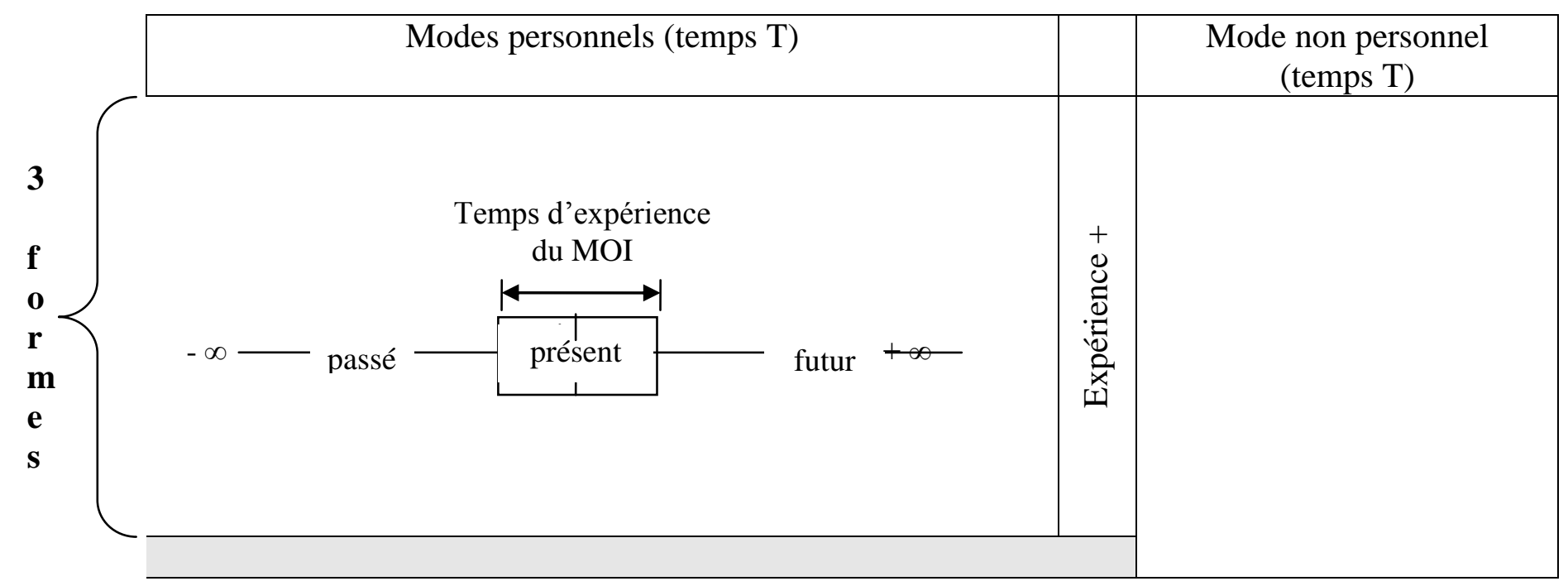




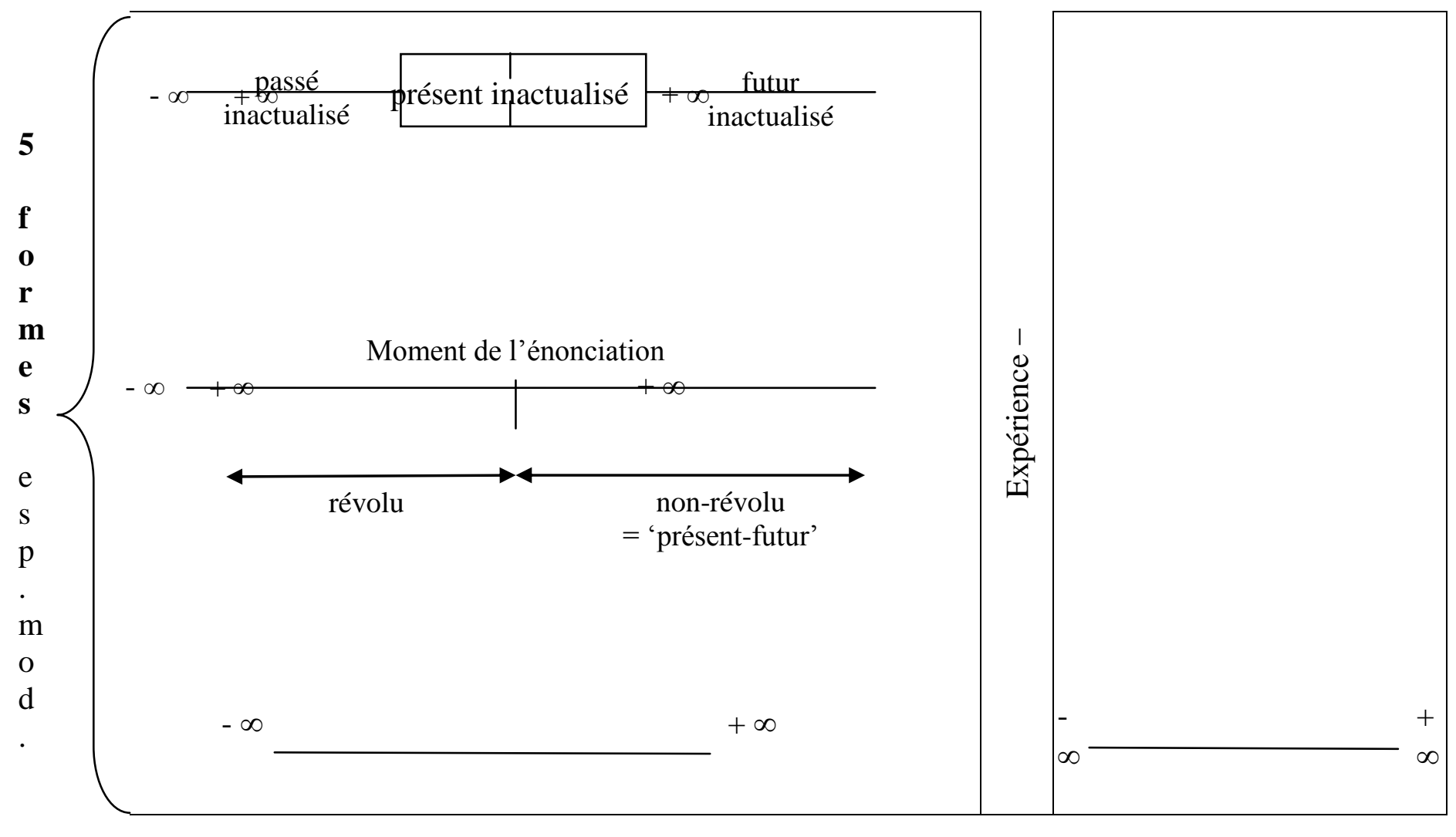

Exochronie +

Exochronie -

Endochronie -

Endochronie +

Le présent d'énonciation, fondateur, se retrouve « la tête en haut ${ }^{9}$, les autres lignes du temps marquant ensuite, les unes après les autres, un éloignement mental par rapport au temps d'expérience du locuteur, au fur et à mesure du paramètre que l'on abandonne : de la vision tripartite qui se dégage de la prise de parole fondatrice du présent inscrit dans l'expérience, on passe à un univers mental marquant le renoncement au présent d'expérience.

Ces représentations temporelles marquent progressivement l'abandon d'un temps divisé, tripartite, ce qui explique que dans le mode inactualisant, les formes verbales soient en plus grand nombre que dans le mode actualisant. Le renoncement à «la tyrannie du MOI » implique donc une certaine complexité du mode inactualisant. La première étape dans le mode inactualisant montre deux formes verbales, l'imparfait et le conditionnel, indifférentes à la division tripartite (passé et présent-futur) mais différenciées par rapport au présent, ici inactualisé, et par rapport au futur, ici inactualisé. L'étape suivante marque l'abandon de ce paramètre en n'ouvrant plus à l'activité du MOI qu'un seul espace délimité à partir du moment d'énonciation, l'espace du non-révolu, lui ôtant, de fait, la possibilité de s'inscrire dans le révolu. Les formes en - $r a$ et en -se, plus abstraites encore que la forme en $-e /-a$, un cran au-dessous dans l'abstraction, sont encore plus

9 D'une certaine façon, cette théorie satisfait le vœu de certains linguistes de voir une «chronogénèse renversée » (De Carvalho 1998: 46) constatant que «beaucoup de tableaux systématique de la psychomécanique ont en quelque sorte la tête en bas » (Roulland 1992 : 184). 
inactualisantes, indifférentes non seulement à l'opposition entre passé, présent et futur, mais aussi à l'opposition entre révolu et non-révolu. L'abandon de ce dernier paramètre aboutit à une représentation du temps de type indivis, où, finalement, l'information sur l'exochronie ne présente aucun intérêt.

\section{Actualité et inactualité locutives}

Le phénomène de la CDT doit être redéfini si l'on prend pour cadre cette nouvelle théorie. À partir du moment où l'on ne raisonne plus en terme d'opposition entre mode indicatif et mode subjonctif, la CDT, posée traditionnellement et uniquement comme concordance inter-modale- mode indicatif dans la principale/mode subjonctif dans la subordonnée -, est caduque et doit laisser place à une redéfinition des relations temporelles dans la phrase complexe ${ }^{10}$.La phrase subordonnée n'est plus le cadre de l'opposition entre le vrai et le non vrai, le réel et le non réel. Elle est le cadre syntaxique où s'exprime pour le locuteur la possibilité de repérer des événements avec les deux modes actualisant/inactualisant en fonction de ce qu'il souhaite ramener à son actualité et ce qu'il souhaite éloigner mentalement de cette actualité.

De fait, il existe deux types de concordances : une concordance intra-modalemise en relation de deux formes verbales du mode inactualisant- et une concordance inter-modale- mise en relation de deux formes verbales, l'une appartenant au mode actualisant, l'autre au mode inactualisant.

\begin{tabular}{|c|c|}
\hline $\begin{array}{c}\text { Concordance inter-modale } \\
\text { Du mode actualisant au mode inactualisant }\end{array}$ & $\begin{array}{c}\text { Concordance intra-modale } \\
\text { À l'intérieur du mode inactualisant }\end{array}$ \\
\hline 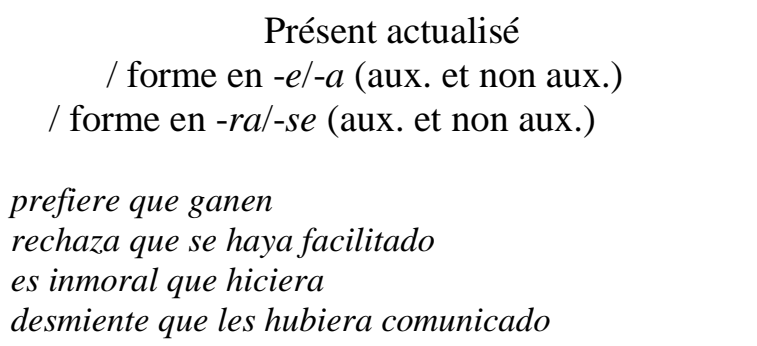 & $\begin{array}{l}\quad \text { Présent inactualisé } \\
\quad \text { / forme en -el-a (aux. et non aux.) } \\
\text { / forme en -ral-se (aux. et non aux.) } \\
\text { no quería que se muestre } \\
\text { negaba que haya cobrado } \\
\text { deseaba que se extendiera } \\
\text { negaba que hubiera sido presionado }\end{array}$ \\
\hline $\begin{array}{l}\text { Présent actualisé d'aspect transcendant } \\
\text { / forme en -el-a (aux. et non aux.) } \\
\quad \text { / forme en -ral-se (aux. et non aux.) } \\
\text { ha apelado a que se mantenga } \\
\text { ha negado que haya participado } \\
\text { ha lamentado que no llegara } \\
\text { ha negado que hubiera tomado }\end{array}$ & $\begin{array}{l}\quad \text { Présent inactualisé d'aspect transcendant } \\
\quad \text { / forme en -el-a (aux. et non aux.) } \\
\quad \text { / forme en -ral-se (aux. et non aux.) } \\
\text { había pedido que sea destruida } \\
\text { había rechazado que haya usado } \\
\text { había aceptado que se terminara } \\
\text { había negado que hubiera escondido }\end{array}$ \\
\hline Futur actualisé & Futur inactualisé \\
\hline
\end{tabular}

${ }^{10}$ Sur la nécessité de « repenser la subordonnée », nous renvoyons à notre ouvrage 2010 : 149-156. 


\begin{tabular}{|c|c|}
\hline $\begin{array}{l}\text { / forme en -el-a (aux. et non aux.) } \\
\quad \text { / forme en ral-se } \\
\text { ordenará que se efectúe } \\
\text { se exigirá que haya cotizado } \\
\text { será posible que nadie viera }\end{array}$ & $\begin{array}{l}\text { / forme en -el-a (aux. et non aux.) } \\
\text { / forme en -ral-se } \\
\text { sería necesario que se incrementen } \\
\text { exigiría que haya entrado } \\
\text { convendría que se investigara }\end{array}$ \\
\hline $\begin{array}{l}\text { Futur actualisé d'aspect transcendant } \\
\text { / forme en -el-a } \\
\text { / forme en -ral-se } \\
\text { te habrá sorprendido que me dirija } \\
\text { habrán deseado que alcanzara }\end{array}$ & $\begin{array}{l}\text { Futur inactualisé d'aspect transcendant } \\
\text { / forme en -el-a (aux. et non aux.) } \\
\text { / forme en -ra/se (aux. et non aux.) } \\
\text { le habría gustado que esté presidida } \\
\text { habría ocasionado que haya ido a pique } \\
\text { le habría gustado que se publicaran } \\
\text { nos habría gustado que hubiera sido recogido }\end{array}$ \\
\hline 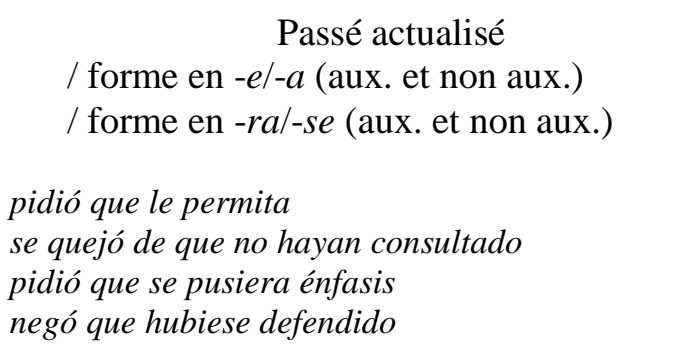 & $\begin{array}{l}\text { Forme en }-e /-a \\
\text { / forme en }-e l-a \\
\text { / forme en -ra } \\
\text { supongamos que sea verdad } \\
\text { imaginemos que estuviera gobernada }\end{array}$ \\
\hline $\begin{array}{l}\text { Passé actualisé d'aspect transcendant } \\
\text { / forme en -ra } \\
\text { le hubo pedido que se apresurara }\end{array}$ & $\begin{array}{l}\text { Forme en -ra } \\
\text { / forme en -el-a } \\
\text { / forme en -ral-se (aux. et non aux.) } \\
\text { quisiera que abandone la isla } \\
\text { quisiera que estuvieran encaminadas } \\
\text { quisiera que hubiese sucedido }\end{array}$ \\
\hline & $\begin{array}{l}\text { Forme en -ra d'aspect transcendant } \\
\text { / forme en -el-a (aux. et non aux.) } \\
\text { / forme en -ra } \\
\text { le hubiera pedido que se postule } \\
\text { me hubiera gustado que se haya plasmado } \\
\text { no hubiera evitado que perdiéramos }\end{array}$ \\
\hline
\end{tabular}

Le locuteur hispanophone a deux choix à partir de son présent d'énonciation :

-il peut tout inactualiser. Principale et subordonnée sont au mode inactualisant : c'est une concordance intra-modale avec, éventuellement, des degrés dans l'inactualité : no quería que se muestre sería necesario que se incrementen convendría que se investigara... 
- Il peut actualiser et inactualiser. La principale est au mode actualisant, la subordonnée au mode inactualisant. C'est une concordance inter-modale avec, éventuellement, des degrés dans l'inactualité : prefiere que ganen negó que hubiese defendido pidió que se pusiera énfasis...

7. Veinticuatro horas después del atentado, Shihaz, de 20 años, estudiante de químicas en la Universidad de A Najah, es una desconocida. Ninguno de los grupos radicales palestinos ha reivindicado la autoría de la operación, ni ha hecho sobre ella el panegírico que habitualmente suele emitirse sobre los que mueren en operaciones similares. Más preocupante aún, los servicios de seguridad de ambos lados dudan de que éste sea su verdadero nombre o de que fuera estudiante en activo de esta universidad, situada en Nablús y considerada como uno de los principales baluartes de Hamás en Cisjordania. (El País, 29-01-02)

Dans cet exemple, le journaliste rapporte un double doute sur la jeune femme auteur de l'attentat-suicide, introduit par le verbe « dudan » au présent actualisé : doute sur l'identité et doute sur son statut d'étudiante. L'emploi de la forme en -ra permet au journaliste de hiérarchiser ces deux inconnues : il n'est pas sûr pour les enquêteurs que son nom soit le bon, ce qui n'a rien d'étonnant dans le cas d'une terroriste ; ce premier doute est simplement exprimé avec la forme en -e/-a. Mais à ce doute vient s'en ajouter un autre : contrairement aux informations dont disposent les autorités, il est encore moins sûr qu'elle appartienne à cette université, connue pour abriter des membres du Hamas, puisque l'organisation n'a pas revendiqué l'attentat. Les événements signifiés par «sea » et «fuera »sont tout simplement hiérarchisés dans le domaine de l'improbable : il est peu probable que le nom évoqué soit le bon ; il est encore plus improbable que la jeune femme ait été une étudiante de l'université en question. L'événement le plus improbable est exprimé sous une forme qui le détache totalement de l'actualité, la forme en ra.

8. [el arzobispo de Córdoba] puntualizó que lo que buscan los obispos es "alertar sobre la necesidad de una coparticipación equilibrada en la cual también los costos que hay que pagar sean distribuidos según las posibilidades de cada uno y no recaigan sobre los que tienen menos capacidad de defensa".

Tras señalar que "todosquisiéramosque hubieramenos pobreza, que hubiese más trabajo, que hayajubilados que se les devuelva lo que aportaron y no jubilados entre comillas", dijo que "éstos son problemas que existen y que los obispos no pueden negar". Añadió en ese sentido que "el gobierno tendrá que dar soluciones integrales" a esas carencias, las que "buscará con seriedad y con las limitaciones que pueda tener como cualquier sector", pero aclaró que a los obispos no les compete abrir juicios "sobre aspectos técnicos de la economía".(La Prensa28/04/1992)

Cet exemple est une illustration assez remarquable de ce qu'il faut entendre par « degrés de l'inactuel ». Certes, le choix de la forme en -ra pour le verbe introducteur, "quisiéramos », laisse entendre que vont s'exprimer des vœux pieux, voués fatalement à l'inactuel ; néanmoins, ces vœux font l'objet d'un traitement différencié, selon que le souhait est rattaché à la notion la plus abstraite dans un propos idéalement humaniste mais totalement irréalisable, « que hubiera menos pobreza » (exprimé par la forme la plus inactualisante), ou qu'il exprime une préoccupation laissant entrevoir une possibilité d'intervention et d'amélioration, un souhait conçu comme moins irréel - justifiant le choix de la 
forme inactuelle en -se, «que hubiese más trabajo »-, ou qu'enfin il exprime une réelle demande de voir s'améliorer le sort des retraités, « que hayajubilados que se les devuelva lo que aportaron ». Ici, le choix de la forme en -e/-a ramène le propos dans le débat socio-économique de l'actualité évoquée plus haut (« una coparticipación equilibrada en la cual también los costos que hay que pagar sean distribuidos según las posibilidades de cada uno...»), et rattachée à la réalité argentine la plus brûlante, celle des inégalités sociales.

9. Algún portavoz de algún partido parlamentario, incluso algún ministro, maldijeron la hora en que José María Aznar, durante el pasado debate sobre el Estado de la Nación, solicitóque se investigara el "caso Filesa" y Felipe González le replicó que también se investigue el "caso Naseiro", lo que acabó por arreglar Miguel Roca, por evitar un enfrentamiento interno en el Grupo Socialista, cuando añadió el "etcétera". (El Mundo 03-10-94)

Dans cet exemple, le jeu entre «se investigara » et «se investigue » permet au journaliste qui rapporte l'échange de marquer l'attaque d'Aznar, première mais néanmoins prudente, avec la forme la plus inactualisante «se investigara », et la défense de González, seconde mais néanmoins menaçante « se investigue ». Il y a, d'une certaine façon, match nul en terme de coups portés, de « cas » à régler, mais le journaliste peut rendre compte de l'escalade verbale entre les deux adversaires.

10. Y resulta que [el seleccionador nacional de fútbol] Bielsa no es el más piola de la cuadra pero tampoco uno del montón. No será casual que más de cuatro clubes poderosos aspiraran a seducirlo y otros tantos esperen el momento adecuado. No es casual que se lo esté requiriendo para conducir a la selección de un país donde los argentinos no gozan de cariños copiosos. Bielsa tiene mucho para dar. (Olé 05-08-07)

Les deux formes verbales, «aspiraran » et «esperen »sont liées à une visée perspectivante : l'envie de recruter Bielsa. Mais le journaliste marque le contraste entre les deux démarches de recrutement : tandis que l'une reste dans l'imaginaire «aspiraran a seducirlo », l'autre apparaît plus concrète et laisse présager une démarche précise et opportuniste, « esperen el momento adecuado ».

\section{Conclusion}

La CDT, pour un locuteur hispanophone, ne se définit ni en terme de temps d'événements, ni en terme uniquement de concordance inter-modale ou intramodale, mais en terme d'occupation d'espaces temporels. C'est la raison pour laquelle il est absolument crucial de poser au préalable une théorie sur le représenté temporel des formes verbales espagnoles pour ensuite rendre compte des concordances d'espaces temporels effectivement rendues possibles par la langue, et concrètement exploitables par le locuteur pour sa visée de discours. 
Moteurs de recherche

\section{Références}

www.elpais.com

www.abc.es

www.eluniversal.com.mx

DAVIS Mark, Corpus del español, www.corpusdelespañol.org

REAL ACADEMIA ESPAÑOLA, Corpus de Referencia del Español Actual (CREA), www.rae.es

ALCINA FRANCH J. \& BLECUA J.M. (2001) (1 éd.1975), Gramática española, Barcelona : Ariel.

BARRALM. (1980), L'imparfait du subjonctif. Étude sur l'emploi et la concordance des temps du subjonctif, Paris : Picard.

BELLO A. (2004) (1 éd.1847), Gramática de la lengua castellana destinada al uso de los americanos, Madrid: Edaf Editorial.

BEDEL J.M.(1997), Grammaire de l'espagnol moderne, Paris : PUF.

BENVENISTE É. (1970), «Le langage et l'expérience humaine», Problèmes $d u$ langage, Paris : Gallimard, 3-13.

BOUZET J. (1984), Grammaire espagnole, Paris : Belin.

BRUNOT F. (1965), La pensée et la langue, Paris : Masson et $C^{\text {ie }}$

CALL DAVI R.(2008), «Aproximación al estudio diacrónico de la consecutio temporum », inRomero Aguilera, L. \&Juliá Luna, C. (coords.), Tendencias actuales enla investigación diacrónica de la lengua-Actas del VIII Congreso Nacional de la Asociación de Jóvenes Investigadores de Historiografía e Historia de la Lengua Española (AJIHLE), Barcelona, del 2 al 4 de abril de 2008: Universitat de Barcelona, 217-225.

CANO AGUILAR R.(1981), Estructuras sintácticas transitivas en el español actual, Madrid : Gredos.

CARRASCO GUTIÉRREZ Á. (1999), «El tiempo verbal y la sintaxis oracional. La consecutio temporum », inBosque, I. \& Demonte, V. (eds.), Gramática descriptiva de la Lengua Española, Madrid : Espasa Calpe, 3061-3128.

CARRASCO GUTIÉRREZ Á.(1994), «La concordancia de tiempos en las gramáticas del español », Verba, 21, 113-131.

CARRASCO GUTIÉRREZÁ. (2000), La concordancia de tiempos, Madrid: Arco/Libros.

CARRASCO GUTIÉRREZÁ.\& GARCÍA FERNÁNDEZ L., «Observaciones sobre la correlación de tiempos", inWotjak, G. (ed.), El verbo español. Aspectos morfosintácticos, sociolingüísticos y lexicogenéticos, Franckfurt am Main : Vervuert, 6171.

CHEVALIER J.-C. (1982), «Le péché de réalité », Langues et linguistique, $\mathrm{n}^{\circ}$ 8, t. 2, 92-125.

CHEVALIER J.-C. (1996), «De Guillaume à la linguistique du signifiant », Modèles Linguistiques, t. XVII, vol. 33, 77-92.

CHEVALIER J.-C. \& DELPORT M.-F. (1995), «Temps des choses, temps de l'outil », Modèles Linguistiques, Temps et langage (I), t. XVI, fasc.1, 149-164.

COMRIE B.(1986), « Tense in Indirect Speech », Folia Linguistica, XX, 265-296.

DE CARVALHO P.(1998), «Subjonctif et chronogénèse en morphosyntaxe comparée », Modèles Linguistiques, t. XIX, fasc.1, 39-59.

COSTE J. \& REDONDO A. (1986), Syntaxe de l'espagnol moderne, Paris : Sedes. 
DELBECQUE N. \& LAMIROY B. (1999), «La subordinación sustantiva: las subordinadas enunciativas en los complementos verbales », inBosque, I. \& Demonte, V. (eds.), Gramática descriptiva de la Lengua Española, Madrid : Espasa Calpe, 1965-2081. DORTA LUIS J.(1987), Modos y tiempos del verbo en la tradición gramatical hispánica desde Nebrija hasta Bello (1492-1860), Tesis doctoral, Islas Canarias :Universidad La Laguna.

ENÇ M. (1987), « Anchoring Conditions for Tense », Linguistic Inquiry, 18.4, 633-657.

ERNOUT A. \& THOMAS F. (2002) (1 éd.1951), Syntaxe latine, Paris : Klincksieck.

FARLEY R. (1965), « Sequence of Tenses: a Useful Principle? », Hispania, 48, 3, 549553.

FARLEY R. (1970), « Time and the Subjunctive in Contemporary Spanish », Hispania, $53,3,466-475$.

FEUILLETJ. (1992), «Typologie de la subordination », Subordination-Subordinations.

Travaux linguistiques du CERLICO, Rennes : Presses Universitaires de Rennes.

FEYDIT F.(1953), «Concordance des temps », Le français moderne, 15, 275-280.

GERBOIN P. \&LEROY C. (1994), Grammaire d'usage de l'espagnol contemporain Paris : Hachette.

GILI GAYA S.(1989), Curso superior de sintaxis española, Barcelona : Vox.

HAJRULLAU B. (2007), La concordance des temps dans les langues romanes. Une spécificité de l'italien : l'expression du futur dans le passé, Thèse de doctorat (Université Paris-Sorbonne)

JOLY A.(1995), «De quelques constantes dans la représentation cognitive et linguistique du temps », Modèles Linguistiques,Temps et langage (I), t. XVI, 27-52.

JOLY A. (2008), "Notes de lecture: de quelques malentendus sur la définition du verbe », Modèles Linguistiques, t. XXIX, vol. 57, 139-149.

KANY C. E. (1969), Sintaxis Hispanoamericana, Madrid : Gredos. Traduction espagnole par M. Blanco Álvarez de l'ouvrage original (1945), American Spanish Syntax, Chicago : University of Chicago Press.

KENINSTON H.(1937), Syntax of the castillian prose, Chicago : University of Chicago Press.

KISHI D. (2004), «Algunas reflexiones sobre la concordancia de los tiempos verbales en el lenguaje periodístico de España, México y Perú », México y la Cuenca del Pacífico, vol.7, $\mathrm{n}^{\circ} 23,138-142$. http://publicaciones.cucsh.udg.mx/pperiod/pacifico/index.html

KOWAL, J. (2007), Spanish Consecutio Temporum: Myths and Reality, Munich : Lincom-Europa.

LAPAIRE J.-R.(1995), "Le temps et sa représentation chez les grammairiens anglophones de Sweet à Langacker », Modèles Linguistiques, t. XVI, fasc.1, 53-69.

LAPESA R., (1969), « La ruptura de la "consecutio temporum” en Bernal Díaz del Castillo », Anuario de letras. Homenaje a Menéndez Pidal, VII,73-83.

LAUNAY M. (1986), «Effet de sens...produit de quoi ?», Langages, n82, 13-37.

LIGATTO D. \& SALAZAR B.(1993), Grammaire de l'espagnol courant, Paris-MilanBarcelone : Masson.

LE TALLEC-LLORET G. (2010a), «La concordance des temps : question de méthode, les méthodes en question », in G. Luquet (éd.), Actes du colloque sur la Concordance des Temps, SEMH-Sorbonne-GERLHIS, 30-31 mai 2008, Colegio de España, Paris : Presses Sorbonne Nouvelle, 63-81.

LE TALLEC-LLORET G. (2010b), La concordance des temps en espagnol moderne. Unité du signe, modes, subordination, Rennes : Presses Universitaires de Rennes.

LE TALLEC-LLORET G. (2011), «Linguistique du signe, linguistique du signifiant : de Mo.La.Che à la cognématique », in G. Luquet (éd.), Morphosytaxe et sémantique espagnoles. Théorie et applications, Paris : Presses Sorbonne Nouvelle, sous presse. 
LUQUET G. (1998), «Peut-on satisfaire aux exigences du signifiant dans une systématique du subjonctif espagnol ? », Modèles Linguistiques, t. XIX, fasc. 1, 89-97. LUQUET G. (2004), La teoría de los modos en la descripción del verbo español. Un nuevo planteamiento, Madrid : Arco/Libros.

LUQUET G. (2006), «Le signifié de langue en tant que représentation différentielle : le cas des formes verbales inactualisantes », in $\mathrm{G}$. Luquet (éd.), Le signifié de langue en espagnol, Paris : Presses Sorbonne Nouvelle, 105-113.

LUQUET G. (2007), « Temps linguistique et "temps verbaux" en grammaire espagnole », LesLangues modernes, 2, 43-58.

LUQUET G. (2008), "Del origen de una equivalencia expresiva de tres términos: hubiera cantado, hubiese cantado, habría cantado, Actas del VII Congreso Internacional de Historia de la Lengua Española, Madrid : Arco/Libros, 837-844.

LUQUET G. (2010), «De l'iconicité des morphèmes grammaticaux en espagnol », in G. Le Tallec-Lloret (éd.), Vues et contrevues, Actes du XII ${ }^{\mathrm{e}}$ Colloque international de linguistique ibéro-romane (LIBERO), Rennes 2, 24-26 sept. 2008, Limoges : LambertLucas, 73-83.

LUQUET G. (2010), «La concordance des "temps" ? Soit. Mais des temps de quels modes ? », in G. Luquet (éd.), Actes du colloque sur la Concordance des Temps, SEMHSorbonne-GERLHIS, 30-31 mai 2008, Colegio de España, Paris : Presses Sorbonne Nouvelle, 83-93.

LUQUET G. \&FERRERES MASPLA, F. (1990), Subjonctif et grammaire systématique française, Barcelone : PPU.

MARCHELLO-NIZIA C. \& PETIOT G. (1972), «La norme et les grammaires scolaires », Langue Française, ${ }^{\circ} 16,99-113$.

MOLHO M.(1975), Sistemática del verboespañol(Aspectos, modos, tiempos), Madrid: Gredos.

MOLHO M., LAUNAY M., CHEVALIER J.-C. (Mo.La.Che)(1984), «La raison du signifiant », Modèles Linguistiques, t. VI, 2, 27-41.

MOLHO M., LAUNAY M., CHEVALIER J.-C. (Mo.La.Che)(1986), «Pour une linguistique du signifiant », Actes du colloque de linguistique hispanique (Rouen, $1^{\mathrm{er}}-2$ février 1985), Cahiers du CRIAR, 6, 95-99.

MOLHO M., LAUNAY M., CHEVALIER J.-C. (Mo.La.Che)(1986), «Le fardeau », Langages, 82, 5-11.

MOESCHLER J. (dir.) (1998),Le temps des événements-Pragmatique de la référence temporelle, Paris : Kimé.

OBAID A.(1967), «A Sequence of Tenses? - What Sequence of Tenses? », Hispania, 50:1, 112-119.

O'KELLY D. (1994), «Du référent expérientiel au référent mental : pour une approche cognitive de la temporalité »,Modèles Linguistiques, vol. 29, t. XV, 1, 25-68.

PARRET H.(1985), «L'oubli naturel des linguistiques du temps », in S. Auroux, J.-C. Chevalier, N. Jacques-Chaquin, C. Marchello-Nizia (dir.), La linguistique fantastique, Paris : Joseph Clims/Denoël, 358-366.

PASQUER C. (2010), "Création et exploitation d'une base de données EXCEL: l'exemple de la Concordance des temps », in G. Le Tallec-Lloret (éd.),Vues et contrevues, Actes du XII ${ }^{\mathrm{e}}$ Colloque international de linguistique ibéro-romane (LIBERO), Rennes 2, 24-26 sept. 2008, Limoges : Lambert-Lucas, 99-108.

RASTIER F. (2007), «Conditions d'une linguistique des normes », in G. Siouffie \& A. Steuckardt (éds.), Les linguistes et la norme : aspects normatifs du discours linguistique, Berne : Peter Lang, 3-20.

REAL ACADEMIA ESPAÑOLA (2004) (1 $1^{\mathrm{e}}$ éd. 1973), Esbozo de una nueva gramática de la lengua española, Madrid : Espasa-Calpe. 
REAL ACADEMIA ESPAÑOLA (2009),Nueva Gramática de la Lengua Española, Vol. 1 « Morphología y Sintaxis », § 24.7 « La concordancia de tiempos. Sus características », Madrid : Espasa Calpe, 1841-1864.

RODGÉ W. (1996), «Valeur fondamentale, valeur centrale et invariant en linguistique », Modèles Linguistiques, L'invariant, t. XVII, fasc. 1, 71-76.

ROJO G. (1973), « Acerca de la temporalidad en el verbo español », Boletín de la Real Academia española, 53, 351-375.

ROJO G. (1976), « La correlación temporal », Verba, vol. 3, 65-89.

ROJO G. \&VEIGA A. (1999), «El tiempo verbal. Los tiempos simples », in I. Bosque \& V. Demonte (eds.), Gramática descriptiva de la Lengua Española, Madrid: Espasa Calpe, 2867-3060.

ROULLAND D. (1992), «La subordination non finie en anglais », Subordination, Travaux linguistiques du CERLICO, Rennes : Presses Universitaires de Rennes, 160-184.

SEGURA RAMOS B. (1974), « La consecutio temporum: una posibilidad de relación temporal », Estudios clásicos, 18, 217-233.

SEGURA RAMOS B. (1978), «La consecutio temporum », Estudios clásicos, 22, 201-211.

SERRALTA F. (1984), "Sur une fausse norme de notre grammaire espagnole», Les langues néo-latines, ${ }^{\circ}$ 250-251, 118-123..

SERRALTA F. (1994), «Une fausse norme qui a la vie dure », Les langues néo-latines, ${ }^{\circ}$ 290, 107-114.

SIOUFFI G. \& STEUCKARDT A. (éds.) (2007), Les linguistes et la norme Aspects normatifs du discours linguistique, Actes des Journées d'étude organisées à Montpellier par l'axe «Diachronie et comparatisme» (EA 739 «Dipralang »), 23 septembre 2003 et 10 avril 2004, Berne : Peter Lang. Publication et données bibliographiques détaillées disponibles sur internet sous http://dnb.ddb.de

SOUTET O.(1998), «La morphologie du subjonctif français: essai de synthèse historique », Modèles Linguistiques, t. XIX, fasc.1, 7-16.

SZNAJDER L.(2002), «La concordance des temps comme paramètre du discours indirect en latin », in L. Savicki \&D. Shalev (éds.), Donum Grammaticum, Studies in Latin and Celtics Linguistics, in honour of Hannah Rosén, Louvain-Paris-Sterling: Peeters, 337-339.

SZNAJDER L. (2006), «À propos des subordonnées complétives latines au subjonctif sans conjoncteur: quelles marques de dépendance grammaticale?», Colloque « Typologie et modélisation de la coordination et de la subordination », (LACITOS-Paris 3, 26-28 mai 2005), Faits de Langue, Coordination et subordination: typologie et modélisation, $\mathrm{n}^{\circ} 28$, Paris : Ophrys.

www.cavi.univ-paris3.fr/ilpga/colloque-coord-subord-2005.

SUNER M. (1979), «El principio de la concordancia temporum en la gramática generativa » Revista de lingüística teórica y aplicada, 17, 7-17.

SUNER M. (1990), «El tiempo en las subordinadas », in Ignacio Bosque (ed.), Tiempo y aspecto en español, Madrid : Cátedra, 77-105.

TOLEDO G. (1987), « Patrones temporales en el español americano », Revista argentina de lingüística, 3, 1, 55-68.

VARGA D.(1998), «Discours indirect dans les langues romanes : la question de la concordance des temps », SRAZ, XLIII, 1-9.

VARGA D. (2000), «Discours indirect dans les langues romanes : les modes », Studia Romanica et Anglica Zagrabiensia (SRAZ), XLV-XLVI, 75-86.

VEIGA A. \&MOSTEIRO LOUZAO M.(2006), El modo verbal en cláusulas condicionales, causales, consecutivas, concesivas, finales y adverbiales de lugar, tiempo y modo, Salamanca : Ediciones Universidad Salamanca. 
VEIGA A.(1996), « De sintaxis temporal española: correlación temporal y cronología relativa de procesos verbales », in M. Casado Velarde etalii (eds.), Scripta Philologica in memoriam Manuel Taboada Cid, La Coruña : Universidad de La Coruña, 737-764.

VEIGA A. (2001), «Te he pedido que vengas: La forma verbal cante y la relación temporal pos-ante-presente », in Bod de Jonge (ed.), Actas del I congreso de la Asociación de Lingüística y Filología de América Latina (ALFAL), Universidad de Groningen, Holanda, vol. 13. http://elies.rediris.es/elies13/veiga.htm

VEIGA A. (2010), « Sistema verba, sintaxis y correlación temporal », in G. Luquet (éd.), Actes du colloque sur la Concordance des Temps, SEMH-Sorbonne-GERLHIS, 30-31 mai 2008, Colegio de España, Paris : Presses Sorbonne Nouvelle, 15-31.

WILMET M. (1995), «L'articulation mode-temps-aspect dans le système verbal français », Modèles Linguistiques, t. XVI, fasc. 1, 91-110.

WOTJAK G. \& Veiga A. (1990), La descripción del verbo español, Santiago de Comostela : Universidad de Santiago de Compostela.

ZAMORANO AGUILAR A. (2005), El subjuntivo en la historia de la gramática española(1771-1973), Madrid : Arco Libros. 